\title{
ESTRATÉGIA EDUCATIVA COM PROFISSIONAIS DE ENFERMAGEM SOBRE HIPERTERMIA MALIGNA EM UM CENTRO CIRÚRGICO CARDIOVASCULAR
}

\author{
Educational strategy with nursing professionals on malignant \\ hypertermia in a cardiovascular surgical center \\ Estrategia educativa con profesionales de enfermería en \\ hipertermia maligna en un centro quirúrgico cardiovascular
}

Elisabete Silvana de Oliveira Sene ${ }^{1 *} \mathbb{\infty}$, Tatiana Garcia Viana ${ }^{2} \mathbb{C}$, Keren Agra Catunda Bettero Valle ${ }^{3} \mathbb{C}$, Eliana Bittar ${ }^{4} \mathbb{C}$

RESUMO: Objetivos: Avaliar o conhecimento da equipe de enfermagem do centro cirúrgico (CC) sobre hipertermia maligna (HM), antes e depois de aula expositiva, e implantar kit para tratamento da HM com os anestesiologistas. Método: Estudo quase experimental do tipo antes e depois. Coleta de dados com 43 profissionais (três enfermeiros, sete técnicos e 33 auxiliares) do CC de um hospital de cardiologia de São Paulo. Aplicaram-se cinco questões antes e depois de aula expositiva, e utilizaram-se teste exato de Fisher e teste de McNemar. Após análise da literatura e dos materiais do setor e reunião com equipe médica, elaborou-se um kit de tratamento. Resultados: Houve diferença significante $(\mathrm{p}<0,05)$ em relação ao conhecimento da equipe sobre HM; após a aula, viu-se progresso nas três categorias (auxiliares $89,42 \%$, técnicos $90 \%$ e enfermeiros 100\%). As questões com porcentagem acima de $90 \%$, após a aula, foram: definição de HM, agentes desencadeantes e tratamento. Foi elaborado e implantado um kit de tratamento. Conclusão: Avaliou-se o conhecimento da equipe de enfermagem sobre HM, antes e depois da aula expositiva, e implantou-se kit de tratamento. O conhecimento da equipe foi satisfatório após a aula ministrada. Palavras-chave: Equipe de enfermagem. Hipertermia maligna. Enfermagem de centro cirúrgico. Enfermagem perioperatória.

ABSTRACT: Objectives: To evaluate the knowledge of the nursing staff of the surgical center (SC) about malignant hyperthermia (MH), before and after lecture, and to implant a kit for the treatment of MH with anesthesiologists. Method: Quasi-experimental before and after study. Data collection with 43 professionals (three nurses, seven technicians and 33 assistants) from the SC of a cardiology hospital in São Paulo. Five questions were applied before and after lecture, and McNemar and Fisher's exact tests were used. After analyzing the literature and industry materials and meeting with the medical team, a treatment kit was prepared. Results: There was a significant difference $(\mathrm{p}<0.05)$ regarding the team's knowledge about MH; After class, progress was seen in the three categories (assistants $89.42 \%$, technicians $90 \%$, and nurses $100 \%$ ). The questions with percentage above $90 \%$ after class were: definition of $\mathrm{MH}$, triggering agents, and treatment. A treatment kit was developed and implemented. Conclusion: The knowledge of the nursing staff about MH before and after the lecture was evaluated, and a treatment kit was implemented. The knowledge of the team was satisfactory after the taught class. Keywords: Nursing, team. Malignant hyperthermia. Operating room nursing. Perioperative nursing.

RESUMEN: Objetivos: Evaluar el conocimiento del personal de enfermería del quirófano (CQ) sobre la hipertermia maligna (HM), antes y después de la conferencia, e implantar un kit para el tratamiento de HM con anestesiólogos. Método: Cuasi-experimental antes y después del estudio. Recopilación de datos con 43 profesionales (tres enfermeras, siete técnicos y 33 asistentes) del CQ de un hospital de cardiología en São Paulo. Se aplicaron cinco preguntas

'Enfermeira; especialista em Centro Cirúrgico, Recuperação Anestésica e Centro de Materiais e Esterilização (CC/RA/CME) pela Faculdade Israelita de Ciências em Saúde Albert Einstein (FICSAE) - São Paulo (SP), Brasil. 2Enfermeira; especialista em Cardiologia pela Universidade Federal de São Paulo (UNIFESP) - São Paulo (SP), Brasil.

${ }^{3}$ Enfermeira; especialista em CC/RA/CME pela FICSAE - São Paulo (SP), Brasil.

¿Enfermeira; doutora em Práticas de Gerenciamento em Enfermagem e em Saúde pela Universidade de São Paulo (USP). Diretora técnica de Serviço de Saúde do Instituto Dante Pazzanese de Cardiologia São Paulo (SP), Brasil.

${ }^{*}$ Autora correspondente: bete_sene@hotmail.com

Recebido: 19/04/2019 - Aprovado: 24/11/2019

DOI: $10.5327 / 21414-4425202000010007$ 
antes y después de la conferencia, y se utilizaron la prueba exacta de Fisher y la prueba de McNemar. Después de analizar la literatura y los materiales de la industria y de reunirse con el equipo médico, se preparó un kit de tratamiento. Resultados: Hubo una diferencia significativa (p<0.05) con respecto al conocimiento del equipo sobre MH; Después de la clase, se observó progreso en las tres categorías (auxiliares $89.42 \%$, técnicos $90 \%$ y enfermeras $100 \%$ ). Las preguntas con un porcentaje superior al 90\% después de la clase fueron: definición de MH, agentes desencadenantes y tratamiento. Se desarrolló e implementó un kit de tratamiento. Conclusión: Se evaluó el conocimiento del personal de enfermería sobre MH antes y después de la conferencia, y se implementó un kit de tratamiento. El conocimiento del equipo fue satisfactorio después de la clase impartida.

Palabras clave: Grupo de enfermería. Hipertermia maligna. Enfermería de quirófano. Enfermería perioperatoria.

\section{INTRODUÇÃO}

A hipertermia maligna (HM) é uma doença manifestada como crise hipermetabólica mediante a exposição de um indivíduo a agentes anestésicos inalatórios, como os do grupo dos halogenados ou a succinilcolina. As reações ocorrem mais frequentemente em homens, e as manifestações clínicas variam. Os sinais mais consistentes são hipercarbia, taquicardia sinusal, rigidez muscular generalizada ou sinal do masseter ${ }^{1}$. Em uma crise de HM, a mutação do gene do receptor rianodina e a exposição aos halogenados e relaxantes musculares despolarizantes conduzem à excessiva liberação de cálcio no citoplasma da fibra muscular, levando a um conjunto de eventos bioquímicos resultantes em sinais clínicos e laboratoriais de HM. Ocorrem produção excessiva de calor proveniente da musculatura esquelética rígida, glicólise anaeróbia, aumento de gás carbônico e do ácido lático, hidrólise de adenosina trifosfato (ATP) e ruptura da fibra muscular ${ }^{2}$. A HM foi descrita na década de $1960 \mathrm{e}$ ocorre em 1:10.000 anestesias gerais em crianças e 1:50.000 anestesias gerais em adultos, afetando, dessa forma, principalmente as crianças ${ }^{3}$.

No Brasil, existe desde 1991 o serviço Hotline, para acompanhar por telefone (+55-11-5575-9873) a progressão dos episódios de HM e prestar assistência. Publicou-se uma investigação de sua atividade no ano de 2009, quando foram estimadas 77 crises anuais ${ }^{4}$. Em uma análise das fichas de notificação dos pacientes com suspeita pessoal ou familiar de HM entre 1997 e 2010, a suscetibilidade foi confirmada em $79,4 \%$ dos 92 parentes investigados com teste de contratura muscular in vitro ${ }^{5}$.

A HM é uma emergência anestésica e existem protocolos com funções explícitas para cada membro da equipe, que devem ser padronizados e orientados para atendimento resolutivo. Seu tratamento consiste em reconhecer e remover os desencadeadores e administrar Dantrolene. A ocorrência dessa síndrome no intraoperatório caracteriza situação de emergência e necessita de tratamento imediato ${ }^{6}$.

Em 2004, a Organização Mundial da Saúde (OMS) lançou a Aliança Mundial para a Segurança do Paciente, visando reduzir danos e definir padrões de segurança ${ }^{7}$.

Portanto, o conhecimento da equipe de enfermagem sobre a fisiopatogenia, as complicações e a forma de atendimento na crise de HM é fundamental à assistência ao paciente com competência e qualidade ${ }^{8}$.

Assim, mediante questionamento quanto ao conhecimento da equipe de enfermagem sobre HM, decidiu-se conduzir uma atividade educativa em serviço e explanar o tema, ressaltando as ações da equipe. Escolheu-se aula expositiva, pelo seu potencial de fortalecer o ensino, com base na compreensão de que as estratégias metodológicas devem desenvolver conhecimentos e habilidades e se ater ao paciente em estado de urgência e emergência?.

Listam-se itens indicados para o tratamento da crise de HM, pela Malignant Hyperthermia Association of the United States (MHAUS) ${ }^{10}$. Ainda, a American Association of Nurse Anesthetists (AANA) afirma que a instituição deve adotar um processo de inspeção do kit de tratamento para HM, com atenção à data de expiração dos itens ${ }^{11}$. Logo, acredita-se que a elaboração e a implementação de um kit para HM otimizem o tratamento. Esse kit é ferramenta crucial para o tratamento do paciente que enfrenta uma crise de HM, facilitando o tratamento imediato da ocorrência pelos profissionais de saúde envolvidos.

\section{OBJETIVOS}

- Avaliar o conhecimento da equipe de enfermagem do centro cirúrgico sobre HM, antes e depois de aula expositiva;

- Implantar um kit padronizado para tratamento da hipertermia maligna com os anestesiologistas. 


\section{MÉTODO}

Trata-se de um estudo quase experimental do tipo antes e depois, no qual foi realizada busca sistemática sobre o conhecimento dos enfermeiros, técnicos e auxiliares de enfermagem atuantes no centro cirúrgico (CC) de um hospital-escola referência em cardiologia da cidade de São Paulo.

A instituição possui três edificações, com o total de 378 leitos, e é considerada um hospital de grande porte. O local de coleta de dados da pesquisa possui nove salas cirúrgicas, sendo uma delas híbrida, e quatro leitos para recuperação anestésica (RA). Fizeram-se 3.725 procedimentos cirúrgicos em 2016.

O quadro de profissionais de enfermagem é composto de cinco enfermeiros, sete técnicos e 34 auxiliares de enfermagem. Desse quantitativo, dois enfermeiros foram excluídos, por serem pesquisadores principais. Houve exclusão de um auxiliar em razão de licença. Com base no cálculo amostral com erro alfa de $0,05 \%$, dos 46 profissionais, inseriram-se no estudo 43 ( $93 \%$ da equipe ativa).

O critério de inclusão contemplou profissionais de enfermagem atuantes diretamente na assistência em CC. Excluíram-se profissionais que não desejaram participar e os que gozavam de férias, licença médica, licença-maternidade ou licença-prêmio durante a coleta de dados.

Oprojeto recebeu aprovação do Comitê de Ética em Pesquisa da instituição sede via Plataforma Brasil Certificado de Apresentação para Apreciação Ética (CAAE) no 55069316.6.0000.5462, em 31 de janeiro de 2016. Os participantes assinaram o Termo de Consentimento Livre e Esclarecido (TCLE) anteriormente à coleta de dados, respeitando os preceitos éticos da Resolução $n^{\circ}$ 466/2012, do Conselho Nacional de Saúde.

A coleta de dados ocorreu entre setembro e outubro de 2016, utilizando-se instrumento construído pelas autoras, fundamentado nas práticas recomendadas pela Association of periOperative Registered Nurses (AORN) $)^{12}$. O instrumento foi validado por duas enfermeiras especialistas em CC e submetido a análise de confiabilidade, por meio do teste alfa de Cronbach. Ele compreendeu as variáveis do perfil sociodemográfico (idade, sexo, tempo de formação profissional e de atuação em CC) e contemplou cinco questões com cinco alternativas objetivas cada uma, das quais apenas uma era a correta.

As questões envolviam conhecimento a respeito da etiologia da doença, fatores desencadeantes, sinais sugestivos da crise, medicamento para o tratamento e atuação da enfermagem. O coeficiente alfa de Cronbach do questionário foi 1 , indicando a confiabilidade do instrumento, cuja aplicação se deu por duas enfermeiras.
Para cada acerto foi atribuído um ponto, e o escore total do teste de conhecimento equivaleu à soma das respostas corretas. Consideraram-se conhecimento sobre o assunto escores acima de $70 \%$. O questionário foi respondido individualmente, no horário de trabalho, e devolvido imediatamente às pesquisadoras, sendo garantido o anonimato. Em seguida, os participantes assistiram à aula expositiva ministrada pelas autoras, com duração de 30 minutos, utilizando-se o programa Microsoft Office PowerPoint $2010^{\circledR}$. Abordaram-se os conceitos de HM, fisiopatologia e de epidemiologia, o histórico, o Decreto $\mathrm{n}^{\circ} 46.601$, de 12 de março de $2002^{13}$, a Resolução n ${ }^{\circ}$ 1.802/2006, do Conselho Federal de Medicina ${ }^{14}$, classificações, manifestações clínicas, tratamento e medidas preventivas. Após uma semana, os colaboradores responderam novamente ao mesmo questionário, para mensuração do aprendizado.

Posteriormente à coleta, os dados foram tabulados em planilha do software Microsoft Office Excel 2010 ${ }^{\circledR}$. A análise foi sistematizada, e levaram-se em conta os escores de três categorias profissionais (enfermeiros, técnicos e auxiliares de enfermagem), e não os escores isolados de cada sujeito. Para cálculo amostral das variáveis relacionadas às características sociodemográficas, as respostas dos testes foram sumarizadas, apresentadas descritivamente e tabeladas por meio de frequência, valores absolutos e médias. Para análise estatística, foram usados o teste exato de Fisher, que comparou as médias antes e depois de cada categoria, e o teste de McNemar, para avaliar as médias de cada questão. O nível de significância adotado foi de $95 \%(\mathrm{p}<0,05)$.

Elaborou-se um kit de tratamento para HM, conforme o Decreto $\mathrm{n}^{\circ} 46.601$, de 12 de março de $2002^{13}$. O processo de construção do kit aconteceu em fases:

- Busca de evidências na literatura;

- Comunicação entre enfermagem e médicos;

- Solicitação da montagem do kit à farmácia;

- Finalização do kit.

Enquanto critérios para composição do kit para tratamento de HM, avaliaram-se os materiais existentes no kit cirúrgico e de anestesia. Verificou-se que a quantidade de compressas do kit cirúrgico atendia à demanda para o paciente em um episódio de HM; portanto, não foram incluídas compressas no kit de HM. Excluiu-se o soro fisiológico resfriado, por causa da impossibilidade de resfriamento - a farmácia do setor disponibiliza 50 unidades de $500 \mathrm{~mL}$ de soro fisiológico resfriado. Quanto ao circuito do equipamento de anestesia, conforme a MHAUS ${ }^{15}$, há quatro possibilidades de manejo, 
entre elas a substituição do circuito adiante da crise. Em razão do volume, esse material foi excluído, havendo reserva na sala de guarda de material inalatório.

A formulação do kit foi validada pelos profissionais: médico-chefe da seção de anestesiologia, médico-chefe da seção de cirurgia, farmacêutico, enfermeira-chefe do setor e autoras da pesquisa. Houve registro formal dessa reunião, que teve duração de 60 minutos, visando padronizar informações e atualizar o protocolo de atendimento de HM.

\section{RESULTADOS}

Participaram da pesquisa 43 colaboradores ( $93 \%$ da equipe ativa), sendo três enfermeiros, sete técnicos e 33 auxiliares de enfermagem. A idade média foi de $45 \pm 10,3$ anos, e prevaleceu o sexo feminino nas três categorias. Quanto ao tempo de formação, a média foi de $18 \pm 8,6$ anos, e a experiência na área de atuação em CC teve média de $13 \pm 8,5$ anos. As características sociodemográficas são apresentadas na Tabela 1.

Pela Tabela 2, verifica-se o nível de conhecimento dos profissionais antes e depois da aula expositiva.

Visualiza-se, na Tabela 2, diferença estatística do conhecimento após aula expositiva sobre HM nas três categorias. $\mathrm{Na}$ categoria auxiliares de enfermagem, a média de acertos no pré-teste foi $14(44,1 \%)$, para $30,4(89,4 \%)$ no pós-teste, atingindo escore maior do que $70 \%$ de acertos apenas no pós-teste. $\mathrm{Na}$ categoria técnicos de enfermagem, a média de acertos no pré-teste foi $1,2(20 \%)$, para 5,4 (90\%) no pós-teste, atingindo escore superior a $70 \%$ de acertos apenas no pós-teste. Na categoria enfermeiros, a média de acertos no pré-teste foi 2,8 (93\%) para $3(100 \%)$ no pós-teste, atingindo o escore superior a $70 \%$ tanto no pré quanto no pós-teste.
No que se refere aos valores estatísticos $(\mathrm{p}<0,05)$, notou-se significância no conhecimento geral dos profissionais nas fases do pré-teste (valor de 0,2 ) e do pós-teste (valor de 0,8 ).

A Tabela 3 demonstra o comparativo geral de acertos das questões antes e depois da aula expositiva.

Por meio da Tabela 3, constata-se diferença porcentual acima de $90 \%$ em três dos cinco itens do conhecimento após

Tabela 1. Características sociodemográficas dos colaboradores participantes do estudo $(n=43)$.

\begin{tabular}{|l|c|c|}
\hline Variáveis & N & $\%$ \\
\hline Faixa etária (anos) & \multicolumn{1}{|c|}{} \\
\hline $21-30$ & 14 & 2,3 \\
\hline $31-40$ & 13 & 32,6 \\
\hline $41-50$ & 10 & 23,2 \\
\hline $51-60$ & 5 & 11,6 \\
\hline $61-70$ & & \\
\hline Sexo & 32 & 74,4 \\
\hline Feminino & 11 & 25,6 \\
\hline Masculino & & \\
\hline Tempo de formação (anos) & 1 & 2,3 \\
\hline$<5$ & 5 & 11,6 \\
\hline 6 a 10 & 21 & 48,8 \\
\hline 11 a 20 & 16 & 37,2 \\
\hline$>20$ & 8 & 18,6 \\
\hline Tempo de atuação no centro cirúrgico (anos) & \\
\hline 1 a 4 & 13 & 30,2 \\
\hline 5 a 10 & 7 & 16,3 \\
\hline 11 a 15 & 15 & 34,9 \\
\hline$>15$ & 43 & 100,0 \\
\hline Total & & \\
\hline
\end{tabular}

Tabela 2. Nível de conhecimento dos colaboradores no pré e no pós-teste, segundo a categoria profissional.

\begin{tabular}{|c|c|c|c|c|c|c|c|c|c|c|c|c|c|c|}
\hline \multirow{3}{*}{ Variáveis } & \multicolumn{4}{|c|}{$\begin{array}{l}\text { Auxiliares de } \\
\text { Enfermagem }\end{array}$} & \multicolumn{4}{|c|}{$\begin{array}{l}\text { Técnicos de } \\
\text { Enfermagem }\end{array}$} & \multicolumn{4}{|c|}{ Enfermeiros } & \multicolumn{2}{|c|}{$\mathbf{p}^{*}$} \\
\hline & \multicolumn{2}{|c|}{ Pré } & \multicolumn{2}{|c|}{ Pós } & \multicolumn{2}{|c|}{ Pré } & \multicolumn{2}{|c|}{ Pós } & \multicolumn{2}{|c|}{ Pré } & \multicolumn{2}{|c|}{ Pós } & \multirow{2}{*}{$\begin{array}{c}\text { Pré } \\
\mathbf{N}\end{array}$} & \multirow{2}{*}{$\begin{array}{c}\text { Pós } \\
\%\end{array}$} \\
\hline & $\mathbf{N}$ & $\%$ & $\mathbf{N}$ & $\%$ & $\mathbf{N}$ & $\%$ & $\mathbf{N}$ & $\%$ & $\mathbf{N}$ & $\%$ & $\mathbf{N}$ & $\%$ & & \\
\hline Definição de hipertermia maligna & 15 & 44,1 & 30 & 88,2 & 2 & 33,3 & 6 & 100 & 3 & 100 & 3 & 100 & 0,1 & 1 \\
\hline Agentes desencadeantes da crise & 18 & 52,9 & 32 & 94,1 & 1 & 16,7 & 6 & 100 & 3 & 100 & 3 & 100 & 0,4 & 1 \\
\hline Manifestações clínicas iniciais & 15 & 44,1 & 31 & 91,2 & 2 & 33,3 & 4 & 66,7 & 3 & 100 & 3 & 100 & 0,1 & 0,1 \\
\hline Tratamento da hipertermia maligna & 13 & 38,2 & 31 & 91,2 & 0 & 0 & 6 & 100 & 3 & 100 & 3 & 100 & 0,0 & 1 \\
\hline Atuação da equipe de enfermagem & 9 & 26,5 & 28 & 82,4 & 1 & 16,7 & 5 & 83,3 & 2 & 66,7 & 3 & 100 & 0,3 & 1 \\
\hline Média & 14 & 44,1 & 30,4 & 89,4 & 1,2 & 20,0 & 5,4 & 90 & 2,8 & 93,3 & 3 & 100 & 0,2 & 0,8 \\
\hline
\end{tabular}

*Teste exato de Fisher. 
Tabela 3. Comparação de médias de acertos do conhecimento segundo itens de avaliação do pré e do pós-teste da atividade educativa. São Paulo, SP, 2019.

\begin{tabular}{|l|c|c|c|c|c|}
\hline \multirow{2}{*}{ Variáveis } & \multicolumn{9}{|c|}{ Conhecimento } & \multicolumn{2}{c|}{$\mathbf{p}^{*}$} \\
\cline { 2 - 6 } & \multicolumn{2}{|c|}{ Pré-teste } & Pós-teste & N \\
\cline { 2 - 7 } & $\mathbf{N}$ & $\%$ & 39 & 90,7 & 0 \\
\hline Definição de hipertermia maligna & 20 & 46,5 & 41 & 95,3 & 0 \\
\hline Agentes desencadeantes da crise & 22 & 51,2 & 38 & 88,4 & 0,6 \\
\hline Manifestações clínicas iniciais & 20 & 46,5 & 40 & 93 & 0 \\
\hline Tratamento da hipertermia maligna & 16 & 37,2 & 36 & 83,7 & 0,1 \\
\hline Atuação equipe de enfermagem & 12 & 27,9 & 38,8 & 90,2 & 0,1 \\
\hline Média & 18 & 39,8 & & & N \\
\hline
\end{tabular}

*Teste de McNemar.

a aula expositiva: agentes desencadeantes da crise $(95,3 \%-$ 41 acertos), tratamento da $\mathrm{HM}(93 \%-40$ acertos $)$ e definição de HM (90,7\% — 39 acertos).

Conforme preconizado cientificamente, a medicação Dantrolene, indicada para tratar HM, foi disponibilizada na quantidade estabelecida para atendimento imediato. Entre a comissão organizadora do kit, atingiu-se o percentual de concordância de $100 \%$ quanto à sua composição. Os insumos foram listados e depositados em uma caixa (Quadro 1) e o kit disponibilizado na farmácia do setor.

Quadro 1. Kit para tratamento de Hipertermia Maligna (HM)

\begin{tabular}{|c|c|}
\hline Quantidade & Medicamentos e Insumos \\
\hline 36 & Dantrolene \\
\hline 01 & Frasco de $500 \mathrm{ml}$ de água destilada \\
\hline 01 & Frasco de $250 \mathrm{ml}$ de bicarbonato de sódio $8,4 \%$ \\
\hline 05 & Ampolas de glicose $50 \%$ de $20 \mathrm{ml}$ \\
\hline 03 & Ampolas de cloreto de cálcio $10 \%$ de $10 \mathrm{ml}$ \\
\hline 01 & Frasco de manitol $20 \%$ \\
\hline 10 & Ampolas de furosemida de $20 \mathrm{mg}$ \\
\hline 01 & Frasco de soro fisiológico $0,9 \%-100 \mathrm{ml}$ \\
\hline 01 & Frasco de insulina regular (na geladeira da farmácia) \\
\hline 01 & Cateter vesical uretral $n^{\circ} 6,8,10,12,16$ \\
\hline 01 & Sonda nasogástrica $n^{\circ} 6,8,10,12,16,18$ \\
\hline 01 & Dispositivo para transfusão de sangue \\
\hline 02 & Equipos de bomba de infusão \\
\hline 02 & Cateteres intravenosos $n^{\circ} 14,16,18,20,22,24$ \\
\hline 01 & Kit monitorização pressão invasiva \\
\hline 05 & Seringas para gasometria arterial \\
\hline 01 & Cateter venoso central adulto e pediátrico \\
\hline
\end{tabular}

\section{DISCUSSÃO}

A capacitação do profissional de enfermagem possibilita melhores chances de sucesso no atendimento e na recuperação do paciente sob uma crise de HM.O êxito da atuação está relacionado a uma equipe preparada, ciente das estratégias exequíveis por base educativa. É fundamental a junção dos múltiplos saberes e da prática para a ressignificação do conhecimento, tendo em vista a constante mudança no processo de ensino-aprendizagem, com busca de profissionais mais críticos, criativos, competentes e preparados para a vida e para o trabalho ${ }^{16}$.

A educação continuada da equipe, por meio da aula expositiva, ajudou a aumentar o nível de conhecimento dos profissionais de enfermagem do CC, já que a educação é um processo amplo, que vê o profissional por todos os ângulos, de forma humanizada, permanente e coerente, e está atenta às necessidades reais dos colaboradores e do ambiente de trabalho ${ }^{12}$.

A literatura relata crescimento do interesse em melhorar a qualidade e a segurança dos pacientes hospitalizados. Para tanto, é necessário haver progressão na revisão dos mecanismos de segurança, a fim de identificar oportunidades de prevenção e eventos potencialmente fatais, com a finalidade de otimizar a resposta perante as situações de crise ${ }^{17}$.

Os resultados no pré-teste da pesquisa evidenciaram conhecimento insuficiente dos técnicos e auxiliares de enfermagem sobre HM, que obtiveram pontuação abaixo de $70 \%$. Para os enfermeiros, a pontuação de acertos foi de $90 \%$, demonstrando maior conhecimento assistencial sobre HM.

Um estudo realizado nos Estados Unidos apontou o pouco conhecimento da enfermagem sobre o tratamento da crise 
de HM. Ressaltou, também, que uma assistência de enfermagem de qualidade agrega componentes essenciais para a melhoria da qualidade e redução de erros, com preparo da equipe de enfermagem, comunicação eficiente e participação dos familiares ${ }^{18}$.

Na presente pesquisa, após a aula expositiva, houve média percentual de acertos considerável no teste de conhecimento, evidenciando a efetividade do conteúdo apresentado. Na categoria enfermeiros, obteve-se média estatística no pré-teste de 2,8 (93,3\%) para $3(100 \%)$ no pós-teste.Não houve, portanto, pontuação baixa o que sugere maior conhecimento a respeito da temática em razão da formação de nível superior. A categoria técnicos de enfermagem obteve média no pré-teste de $1,2(20 \%)$ para 5,4 (90\%) no pós-teste, e a categoria auxiliares de enfermagem, média no pré-teste de $14(44,1 \%)$ para 30,4 (89,4\%) no pós-teste. Considerando-se o questionário designado para um público profissional técnico, o conhecimento sobre HM no pré-teste, abaixo de $70 \%$, foi visto como insatisfatório, demonstrando falta de informação desses profissionais sobre o assunto. Contudo, esses resultados foram modificados positivamente após a aula educativa: $89,4 \%$ de acertos para auxiliares e $90 \%$ para técnicos, o que correspondeu à meta estabelecida pelas pesquisadoras.

$\mathrm{Na}$ análise dos itens que compõem o questionário, houve diferença significante em três dos cinco elementos após a atividade educativa: definição de HM, com média no pré-teste de $20(46,5 \%)$ para $39(90,7 \%)$ no pós-teste; agentes desencadeantes da crise, com média no pré-teste de $22(51,2 \%)$ para 41 (95,3\%) no pós-teste; e tratamento da HM, com média no pré-teste de $16(37,2 \%)$ para 40 (93\%) no pós-teste.

A literatura ressalta que o processo educativo é um compromisso individual que ocorre por meio de mudanças de atitude em decorrência das experiências vividas nas relações interpessoais sociais e profissionais e que objetiva a transformação da qualificação dos profissionais, para que atuem com mais segurança e rapidez no atendimento ao paciente. O desenvolvimento pessoal que deve ser potencializado caracteriza a educação permanente, envolvendo capacitação técnica específica e aquisição de novos conhecimentos, conceitos e atitudes. Dessa maneira, a educação permanente é imprescindível na prática hospitalar e indispensável à formação dos trabalhadores, a fim de oferecer qualidade, considerando a vida dos pacientes ${ }^{19}$.

É pertinente enfatizar que a HM é classificada como uma doença de notificação compulsória imediata, regulamentada pela Resolução da Secretaria de Estado da Saúde SS-20 e instituída pelo estado de São Paulo no dia 22 de fevereiro de 2006.
Segundo esse documento, o profissional de saúde tem o dever de preencher a Ficha de Notificação Compulsória de Eventos Adversos e encaminhá-la para a farmacovigilância do seu estado ${ }^{20}$.

Na busca da literatura nacional sobre o conhecimento da enfermagem voltado para essa temática, observou-seescassez de material específico que pudesse ser comparado com os achados, denotando a falta de estudos nacionais. Diante disso, é fato a necessidade de treinamento e expansão do conhecimento dos profissionais de saúde para reconhecimento e condução dos pacientes em crise de HM no perioperatório.

Dada a complexidade do tratamento da HM, a literatura sugere o desenvolvimento de dias de treinamento, associado com simulação de incidentes de crise de HM, pelo menos uma vez por ano, com a equipe de enfermagem ${ }^{21}$. Entretanto, a AORN recomenda que o treinamento englobe todos os profissionais do bloco operatório, com foco nas competências aplicáveis às funções necessárias para gerenciar uma crise de $\mathrm{HM}^{22}$. Esses exercícios não apenas mantêm a equipe atualizada e alerta como aumentam a colaboração entre os seus membros. A AANA indica a todos os enfermeiros anestesiologistas certificados que mantenham sua competência por meio da educação continuada no que concerne ao tratamento da HM. A MHAUS salienta a disponibilidade da medicação Dantrolene em todos os estabelecimentos de saúde que realizam procedimentos anestésico-cirúrgicos ${ }^{11}$.

A elaboração e a disponibilização do kit na farmácia do CC com todos os itens necessários para atender ao paciente em uma crise de HM possibilitou a padronização dos materiais imprescindíveis no kit, bem como a atualização e a disponibilização do protocolo de atendimento a toda a equipe do bloco cirúrgico. A padronização é uma importante ferramenta gerencial e confere uniformidade às ações, reduzindo a dispersão e tornando possível a realização dos serviços de forma orientada e segura ${ }^{23}$. O enfermeiro, em sua competência, enfrenta muitos desafios a fim de garantir a segurança do paciente. Assim, os quatro pilares da atuação do enfermeiro (pesquisa, ensino, assistência e gestão) são imprescindíveis no empenho e na capacitação dos profissionais ${ }^{24}$.

A disponibilidade de Dantrolene para tratamento de HM nos estabelecimentos de saúde onde se realizam procedimentos anestésico-cirúrgicos é insuficiente para iniciar o tratamento imediato e, em alguns locais, inexistente. Essa conduta vai contra os princípios constitucionais, e a instituição pode ser penalizada pela irregularidade de expor o paciente a um risco iminente. A existência do kit de HM e do protocolo assistencial específico subsidia um atendimento preciso e imediato ao paciente, e todos os profissionais do bloco operatório são protagonistas de suas ações ${ }^{25}$. 
Um estudo desenvolvido em uma instituição privada de São Paulo ${ }^{26}$ cujo objetivo era avaliar o conhecimento e o índice de acertos da equipe de enfermagem que atua no CC e na recuperação anestésica identificou fragilidade no conhecimento da equipe de enfermagem sobre o diagnóstico e o tratamento de HM e ressaltou que tais fragilidades podem impedir a atuação correta na reversão da crise, facilitando erros que possivelmente trarão malefícios aos pacientes.

Diante do exposto, é vital haver capacitação e trabalho em equipe, de modo a garantir a qualidade da assistência prestada ao paciente em crise de HM, com constante avaliação dessa qualidade, assegurando direitos, segurança e bem-estar do paciente.

Como limitação da pesquisa, citamos os resultados de uma população local, que não podem ser generalizados. A escassez de pesquisas impede um comparativo dos resultados deste estudo com uma população similar. Assim, incentiva-se a realização de pesquisas científicas sobre HM que examinam a atuação da equipe de enfermagem, a fim de expandir o tema, alertar e capacitar os profissionais para atender a esses pacientes com excelência.

\section{CONCLUSÃO}

A crise de HM é um evento grave que exige uma equipe preparada e capacitada para agir e requer diagnóstico, tratamento e assistência imediata que permitam reverter o quadro e o risco de morte. Por ser uma doença de extrema relevância, surgiu inquietação referente ao conhecimento da equipe de enfermagem sobre a doença e à disponibilidade de um kit na instituição para tratamento, conforme recomendação do Ministério da Saúde.

A aula expositiva demonstrou eficácia e agregou conhecimento aos enfermeiros, técnicos e auxiliares da equipe do bloco cirúrgico, o que se mensurou pelos resultados, direcionando os profissionais para atuar com segurança. A existência de um kit para crise de HM é fundamental para garantir o atendimento ao paciente. O conhecimento da equipe acerca da disponibilidade do kit e o reconhecimento das ações dos seus membros perante a crise favoreceram a padronização dos atos designados a cada profissional. Salientamos que um treinamento da equipe com simulação realística agregará expertise no atendimento ao paciente em crise de HM.

Ao enfermeiro, como líder, faz-se essencial atualizar-se cientificamente para uma prática com excelência e expandir o conhecimento de sua equipe, com o objetivo de elevar a competência dos colaboradores. A atuação da equipe de enfermagem, seja na assistência, seja na gestão, em consonância com outros membros da equipe cirúrgica e anestésica, é fator indispensável no momento de auxiliar no reconhecimento e nas manifestações clínicas de uma crise de HM e iniciar as ações necessárias para um atendimento seguro e eficaz.

\section{REFERÊNCIAS}

1. Litman RS. Malignant hyperthermia: diagnosis and management of acute crisis [Internet]. Oxford: UpToDate Database; 2019 [acessado em 26 jul. 2019]. Disponível em: https://www.uptodate.com/contents/ malignant-hyperthermia-clinical-diagnosis-and-management-of-acutecrisis?search=Malignant\%20hyperthermia:\%20clinical\%20diagnosis $\% 20$ and $\% 20$ management $\% 20$ of $\% 20$ acute $\% 20$ crisis $\% 20 \&$ source=search_. result\&selectedTitle=1 150\&usage_type=default\&display_rank=1

2. Silva HCA. Fisiopatologia e genética. Rev Neurociênc [Internet]. 2014 [acessado em 26 jul. 2019];22(4):475-97. Disponivel em: http:// www.revistaneurociencias.com.br/edicoes/2014/2204/VI_curso_ HIPERTERMIA\%2OVOL\%2022\%2004\%202014.pdf

3. Haluka J. Malignant hyperthermia. [Internet]. ACLS Training Center; 2018 [acessado em 3 jan. 2019]. Disponivel em: https://www.acls. net/malignant-hyperthermia.htm
4. Silva HCA, Almeida CS, Brandão JCM, Silva CAN, Lorenzo MEP, Ferreira CBND, et al. Malignant hyperthermia in Brazil: analysis of hotline activity in 2009. Rev Bras Anestesiol [Internet]. 2013 [acessado em 21 jul. 2016];63(1):13-26. Disponível em: http://doi.org/10.1590/ S0034-70942013000100002

5. Silva HCA, Ferreira G, Rodrigues G, dos Santos JM, Andrade PV, Hortense A, et al. Profile of malignant hyperthermia susceptibility reports confirmed with muscular contracture test in Brazil. Braz J Anesthesiol [Internet]. 2019 [acessado em 25 jul. 2019];69(2):1529. Disponível em: http://doi.org/10.1016/j.bjan.2018.09.007

6. Sousa CS, Bispo DM, Cunha ALM, Siqueira ILCP. Educational intervention on malignant hyperthermia with nursing professionals of the operating room. Rev Esc Enferm USP. 2015;49(2):290-5. http:// doi.org/10.1590/S0080-623420150000200015 
7. Pancieri PA, Carvalho R, Braga ME. Applying the safe surgery checklist: experience report. Rev SOBECC. 2014;19(1):26-33. http:// doi.org/10.4322/sobecc.2014.006

8. Denholm BG. Using a vulnerability theoretical model to assess the malignant hyperthermia susceptible population: implications for advanced practice emergency nurses. Adv Emerg Nurs J [Internet]. 2015 [accessado em 21 mar. 2019];37(3):209-22. http://doi.org/10.1097/ TME. 0000000000000073

9. Morais Filho LA, Gue Martini J, Lazzari DD, Vargas MA, Backes VMS, Farias GM. Strategies used for teaching urgency/emergency in a nursing undergraduate course. Texto \& Contexto Enferm. 2018;27(4):e3210016. http://doi.org/10.1590/0104-07072018003210016

10. Malignant Hyperthermia Association of the United States. What should be on an MH cart? [Internet]. 1981 [acessado em 26 jul. 2019]. Disponível em: https://www.mhaus.org/healthcare-professionals/ be-prepared/what-should-be-on-an-mh-cart/

11. American Association of Nurse Anesthetists. Malignant hyperthermia crisis preparedness and treatment. [Internet]. American Association of Nurse Anesthetists [acessado em 26 jul. 2019]. Disponivel em: https://www.aana.com/docs/default-source/practice-aana-com-webdocuments-(all)/malignant-hyperthermia-crisis-preparedness-andtreatment.pdf?sfvrsn=630049b1_8

12. Cain CL, Riess ML, Gettrust L, Novalija J. Malignant hyperthermia crisis: optimizing patient outcomes through simulation and interdisciplinary collaboration. AORN J. 2014;99(2):301-11. http://doi.org/10.1016/j. aorn.2013.06.012

13. São Paulo (Estado). Secretaria de Saúde do Estado de São Paulo. Resolução SS n 23, de 27 de fevereiro de 2004. Aprova a norma técnica relativa às diretrizes para o diagnóstico, tratamento, prevenção, notificação e investigação epidemiológica dos casos de hipertermia maligna. [Internet]. São Paulo; 2004 [acessado em 28 maio 2017]. Disponível em: http://tc-legis2.bvs.br/leisref/public/showAct. php?id=69\&word $=$

14. Conselho Federal de Medicina. Resolução CFM n 1.802/2006. Dispõe sobre a prática do ato anestésico [Internet]. Brasília: Conselho Federal de Medicina; 2006 [acessado em 31 jul. 2019]. Disponível em: http://www.portalmedico.org.br/resolucoes/ cfm/2006/1802_2006.htm

15. Malignant Hyperthermia Association of the United States. Table of contents. [Internet]. Malignant Hyperthermia Association of the United States; 2012 [acessado em 29 jul. 2019]. Disponível em: https://www. mhaus.org/mhau001/assets/File/Recommendations\%20with\%20 Table\%20of\%20Contents(1).pdf
16. Gottems LBD, Alvarez AM, Almeida LMWS. Nursing education: quality, innovation and accountability. Rev Bras Enferm. 2014;67(4):501-2. http://dx.doi.org/10.1590/0034-7167.2014670401

17. Taguti PS, Dotti AZ, Araujo KP, Pariz PS, Dias GF, Kauss IA, et al. The performance of a rapid response team in the management of code yellow events at a university hospital. Rev Bras Ter Intensiva [Internet]. 2013 [acessado em 5 set. 2017];25(2):99-105. Disponível em: https://doi.org/10.5935/0103-507X.20130020

18. Denholm GB. Using informatics to improve the care of patients susceptible to malignant hyperthermia. AORN J. 2016;103(4):36474. http://doi.org/10.1016/j.aorn.2016.02.001

19. Pinto JR, Ferreira GSM, Gomes AMA, Ferreira FIS, Aragão AEA, Gomes FMA. Permanent education: reflection in practice nursing hospital. Tempus (Brasilia) [Internet]. 2015 [acessado em 29 set. 2017];9(1):155-65. Disponível em: http://www.tempusactas.unb. br/index.php/tempus/article/download/1699/1409

20. São Paulo (Estado). Secretaria de Saúde do Estado de São Paulo. Resolução SS $n^{\circ} 20$, de 22 de fevereiro de 2006: atualiza a lista de doenças de notificação compulsória no estado de São Paulo. [Internet]. São Paulo; 2006 [acessado em 10 out. 2019]. Disponível em: fehosp.com.br/app/ webroot/files/circulares/2b257b09784b0963399612d26cc914fc.doc

21. Theofanis F. Malignant hyperthermia: nursing implications for care. $\mathrm{Br} \mathrm{J}$ Anaesth Recovery Nurs [Internet]. 2015 [acessado em 12 out. 2017];15(4):157. Disponível em: http://eprints.brighton.ac.uk/14549/1/Malignant.pdf

22. Seifert PC, Wahr JA, Pace M, Cochrane AB, Bagnola AJ. Crisis management of malignant hyperthermia in the OR. AORN J. 2014;100(2):189-202. http://doi.org/10.1016/j.aorn.2014.06.014

23. Sene ESO, Jardim DP. Nursing practice in minimally invasive vídeoassisted cardiac surgery. Rev SOBECC. 2016;21(3):170-7. http://doi. org/10.5327/Z1414-4425201600030008

24. Lima GF, Rinaldi PA, Catunda KA, Bittar E, Bianchi ERF. The role of nurses in the structuring and implementation of a hybrid surgical room. Rev SOBECC. 2014;19(2):110-8. http://doi.org/10.4322/ sobecc. 2014.017

25. Sousa CS, Diniz TRZ, Cunha ALSM. Malignant hyperthermia: proposing a care protocol for surgical centers. J Nurs UFPE On line [Internet]. 2013 [acessado em 29 out. 2017]:7(esp.):6714-8. Disponivel em: http://doi.org/10.5205/reuol.5058-41233-3-SM.071 1 esp201320

26. Titato MMS, Carvalho R. Malignant hyperthermia in the operating room: can the nursing team recognize it and intervene? Rev SOBECC. 2017;22(2):82-9. http://doi.org/10.5327/Z1414-4425201700020005 